Article

\title{
Long Non-Coding RNA Expression in Laser Micro-Dissected Luminal $A$ and Triple Negative Breast Cancer Tissue Samples-A Pilot Study
}

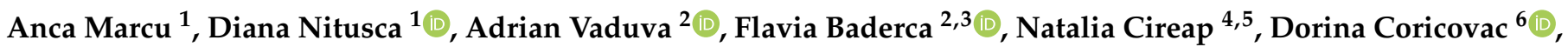 \\ Cristina Adriana Dehelean ${ }^{6}$, Edward Seclaman ${ }^{1}$, Razvan Ilina ${ }^{5, *}$ and Catalin Marian ${ }^{1}$ (D)
}

1 Department of Biochemistry and Pharmacology, Victor Babeş University of Medicine and Pharmacy, Pta Eftimie Murgu Nr.2, 300041 Timişoara, Romania; marcu.anca@umft.ro (A.M.); nitusca.diana@umft.ro (D.N.); eseclaman@umft.ro (E.S.); cmarian@umft.ro (C.M.)

2 Department of Microscopic Morphology, Victor Babeş University of Medicine and Pharmacy, Pta Eftimie Murgu Nr.2, 300041 Timişoara, Romania; vaduva.adrian@umft.ro (A.V.); baderca.flavia@umft.ro (F.B.)

3 Department of Pathology, Emergency City Hospital, 300041 Timişoara, Romania

4 Department of Surgical Oncology, Victor Babeş University of Medicine and Pharmacy, Pta Eftimie Murgu Nr.2, 300041 Timişoara, Romania; nata_cireap@yahoo.com

5 Department of Surgical Oncology, Municipal Hospital, Str. Gheorghe Dima Nr.5, 300254 Timişoara, Romania

6 Faculty of Pharmacy, Victor Babeș University of Medicine and Pharmacy, Pta Eftimie Murgu Nr. 2,

check for updates

Citation: Marcu, A.; Nitusca, D.; Vaduva, A.; Baderca, F.; Cireap, N.; Coricovac, D.; Dehelean, C.A.; Seclaman, E.; Ilina, R.; Marian, C. Long Non-Coding RNA Expression in Laser Micro-Dissected Luminal A and Triple Negative Breast Cancer Tissue Samples-A Pilot Study. Medicina 2021, 57, 371.

https://doi.org/10.3390/medicina 57040371

Academic Editors: Tithi Biswas and Jimmy T. Efird

Received: 16 March 2021

Accepted: 8 April 2021

Published: 12 April 2021

Publisher's Note: MDPI stays neutral with regard to jurisdictional claims in published maps and institutional affiliations.

Copyright: (c) 2021 by the authors. Licensee MDPI, Basel, Switzerland. This article is an open access article distributed under the terms and conditions of the Creative Commons Attribution (CC BY) license (https:/ / creativecommons.org/licenses/by/ $4.0 /)$.
300041 Timişoara, Romania; dorinacoricovac@umft.ro (D.C.); cadehelean@umft.ro (C.A.D.)

* Correspondence: razvanilina@yahoo.co.uk

\begin{abstract}
Background and Objectives: Breast cancer (BC) remains one of the major causes of cancer death in women worldwide. The difficulties in assessing the deep molecular mechanisms involved in this pathology arise from its high complexity and diverse tissue subtypes. Long non-coding RNAs (lncRNAs) were shown to have great tissue specificity, being differentially expressed within the BC tissue subtypes. Materials and Methods: Herein, we performed lncRNA profiling by PCR array in triple negative breast cancer (TNBC) and luminal A tissue samples from 18 BC patients (nine TNBC and nine luminal A), followed by individual validation in BC tissue and cell lines. Tissue samples were previously archived in formalin-fixed paraffin-embedded (FFPE) samples, and the areas of interest were dissected using laser capture microdissection (LCM) technology. Results: Two lncRNAs (OTX2-AS1 and SOX2OT) were differentially expressed in the profiling analysis (fold change of 205.22 and 0.02 , respectively, $p<0.05$ in both cases); however, they did not reach statistical significance in the individual validation measurement $(p>0.05)$ when analyzed with specific individual assays. In addition, GAS5 and NEAT1 lncRNAs were individually assessed as they were previously described in the literature as being associated with BC. GAS5 was significantly downregulated in both TNBC tissues and cell lines compared to luminal A samples, while NEAT1 was significantly downregulated only in TNBC cells vs. luminal A. Conclusions: Therefore, we identified GAS5 lncRNA as having a differential expression in TNBC tissues and cells compared to luminal A, with possible implications in the molecular mechanisms of the TNBC subtype. This proof of principle study also suggests that LCM could be a useful technique for limiting the sample heterogeneity for lncRNA gene expression analysis in BC FFPE tissues. Future studies of larger cohort sizes are needed in order to assess the biomarker potential of lncRNA GAS5 in BC.
\end{abstract}

Keywords: breast cancer; tissue specificity; lncRNA; laser capture microdissection

\section{Introduction}

Breast cancer (BC), a major health concern, is considered to be the second leading cause of cancer death in women worldwide, with an estimate of 276,480 new cases in 2020 in the US alone, accounting for an estimate of 42,170 new deaths [1]. Heterogenous and 
complex by nature, $\mathrm{BC}$ comprises various and distinct patterns, which are classified in groups and subgroups that aid in the treatment standardization and patient care [2].

There are diverse means of classifying BC. A less costly approach provides an approximate classification based on immunohistochemistry (IHC) [3,4]. However, based on the genes expressed in $\mathrm{BC}$ tissues, there are five distinct subtypes: Luminal $\mathrm{A}$, Luminal $\mathrm{B}$, Triple negative (TNBC)/basal-like, HER2-enriched, and Normal-like, each having different patterns and particularities [5].

In the last decades, abundant literature findings indicate a novel approach toward the analysis of nucleic acids and protein metabolites, which could identify such molecules in archived tissue samples [6]. The high-throughput transcriptome data from BC patients indicated mounting evidence of long non-coding RNA (lncRNA) species that are differentially expressed in tumor tissue samples compared to normal ones [7].

LncRNAs are very abundant and diverse RNA transcripts, with lengths of over 200 nucleotides, that are not generally translated into proteins, and that possess various functions, from which some of the biological mechanisms remain to be understood. However, it is now known that there are several types of lncRNA, such as intergenic-, intronic-, bidirectional-, overlapping sense-, antisense lncRNAs, as well as lncRNAs hosted by a microRNA gene/cluster, with roles at different levels (transcriptional, posttranscriptional, and epigenetic). Moreover, reports showed that lncRNAs are involved in various pathophysiological processes, such as cell cycle regulation, chromatin remodeling, histone modifications, as well as gene imprinting, while others were found to interfere with transcription [8]. In cancer, studies showed that lncRNAs interact with several signaling pathways (Akt-, MAP kinase-, Wnt-, MYC signaling pathways), thus mediating cell proliferation, invasion, metastasis, and apoptosis [9].

In BC in particular, lncRNAs tend to reveal a tissue-specific pattern, as they are differentially expressed in the various subtypes of BC tissues. Microarray findings showed a dysregulated lncRNA expression profile in TNBC tissues, which lead to the hypothesis that they may be involved in the progression of this particular type of BC [10]. Furthermore, a wide number of studies indicated a possible link between lncRNAs involved in different molecular subtypes of this malignancy: LOC554202 was firstly discovered to play a role in the aggressive, estrogen receptor negative TNBC subtype [11], while LOC100288637 had the highest correlation with the HER2 positive subtype [12].

However, available literature reports that investigate comparative differential expression of lncRNAs in distinct tissue subtypes of BC are limited, since the majority of study designs include healthy subjects or adjacent non-tumor tissues as control samples [7]. Moreover, researchers have mainly studied the involvement of lncRNAs in BC using either fresh tissue specimens, TNBC cell lines, or archived FFPE tissue samples using regular protocols. As it is well known that $\mathrm{BC}$ is very heterogenous even within the same tissue slide, therefore, we attempted to limit this heterogeneity from our samples by using laser capture microdissection (LCM) for the dissection of our archived formalin-fixed paraffin-embedded (FFPE) specimens. LCM shows to represent a useful technique for BC tissue analysis, as it is capable of isolating desired cell populations from a very diverse tissue slide [13].

Therefore, we analyzed herein the lncRNA differential expression in two BC subtypes, luminal A and TNBC, in LCM-dissected FFPE archived tissue samples, so as to investigate not only information about the potential BC tissue specificity of different lncRNA species as biomarkers for BC but also for the technological aim of proving that LCM could be a powerful technique for isolating tumor tissues of interest for downstream gene expression analysis. Our study design involved a two-step approach: the "profiling" step, which included the analysis of a large lncRNA panel, and the "validation" step, where we analyzed individual lncRNAs, using specific primer sequences for those regions. 


\section{Materials and Methods}

\subsection{Patients' Characteristics and Tumor Samples}

The full description of the study design and patients' characteristics can be found in two of our previous publications $[13,14]$. To summarize, we analyzed 18 breast tumor slides from patients with diagnosed invasive ductal breast carcinoma, who underwent surgical resection at the Department of Surgical Oncology of the Timişoara Municipal Hospital over one year period (2009-2010). We obtained informed consent from all patients before surgery for using their FFPE tumor tissue slides from the Pathology Department's archive. The study was approved by the Ethical Committee of our institution and performed in accordance with the Ethical Standards of the 1964 Declaration of Helsinki and its later amendments.

\subsection{Laser Capture Microdissection (LCM)}

The preserved tumor tissues were cut with a MMI SmartCut Plus System (MMI Molecular Machines \& Industries, Glattbrugg, Switzerland), isolating from the tissue samples only the malignant groups of cells. This method was described as being able to isolate, procure, and analyze desired cell populations, by cutting a specific area of a tissue sample under microscopic visualization. Despite its limitations, LCM proved to be suitable in the application of RNA transcript profiling, as previously described [13].

Briefly, the FFPE samples were sectioned with a microtome at $10 \mu \mathrm{m}$, mounted on slides free of RNase (MMI MembranSlides, MMI, Glattbrugg, Switzerland), and cut with adequate power and focus for UV laser shots with the aforementioned MMI SmartCut Plus System. The areas were captured, pooled, and placed in a microcentrifuge tube (RNA-free), as indicated by the manufacturer (mmi IsolationCap tubes, MMI, Glattbrugg, Switzerland). Typical slide images and a detailed protocol are described in two of our previous publications $[15,16]$.

\subsection{RNA Extraction}

After microdissection, the cut regions were placed into a collection vessel and underwent a deparaffinization step. The deparaffinization technique used the melting protocol due to the small quantity of the samples. Paraffin was melted and cooled, and the solid layer was pierced with a tip, as indicated by the manufacturer for the miRNeasy FFPE kit (Qiagen, Hilden, Germany).

Total RNA was extracted using the same miRNeasy FFPE kit (Qiagen, Germany) according to the manufacturer's instructions, using the spin column technique for RNA purification, which included ethanol precipitation of nucleic acids.

Following extraction, we eluted the samples with $12 \mu \mathrm{L}$ RNase-free water and quantified the RNA concentration with the Qubit RNA HS Assay Kit (ThermoFisher Scientific, Waltham, MA, USA) using a Qubit ${ }^{\circledR}$ Fluorometer.

\subsection{LncRNA Profiling in FFPE Tissues}

Following total RNA extraction, we performed reverse transcription reactions in order to obtain cDNA for the qRT-PCR amplification step. A fixed volume of $8 \mu \mathrm{L}$ from the total RNA was input into subsequent cDNA reactions, using the RT2 PreAMP cDNA Synthesis kit (Qiagen, Germany). This includes a preamplification step in order to improve the low copy number lncRNA detection. The cycle conditions for the preamplification of cDNA from our FFPE tissue samples were selected according to the manufacturer's indications with a $10 \mathrm{~min}\left(95^{\circ} \mathrm{C}\right)$ HotStart DNA Taq Polymerase activation step followed by 8 cycles $\left(95{ }^{\circ} \mathrm{C} / 15 \mathrm{~s}, 60^{\circ} \mathrm{C} / 2 \mathrm{~min}\right.$ ). Quantitative real-time PCR was immediately performed to quantify 84 lncRNAs using the RT ${ }^{2}$ lncRNA PCR Array Human lncFinder (LAHS-001ZE; Qiagen, Germany) combined with $\mathrm{RT}^{2} \mathrm{SYBR}^{\circledR}$ Green qPCR Mastermix (Qiagen, Germany). Each reaction was performed in triplicate, with the following cycle conditions indicated by the protocol: $95^{\circ} \mathrm{C} / 10 \mathrm{~min}(1 \mathrm{cycle}), 95^{\circ} \mathrm{C} / 15 \mathrm{~s}, 60^{\circ} \mathrm{C} / 1 \mathrm{~min}$ ( 40 cycles). 


\subsection{LncRNA Validation}

The differentially expressed lncRNAs (OTX2-AS1 and SOX2OT), together with GAS5 and NEAT1 selected from literature were further validated using TaqMan Fast Advanced Master Mix/TaqMan individual assays, (assay ID Hs01008264_s1 for NEAT1, and Hs03464472_m1 for GAS5, respectively, from Thermo Fisher Scientific, USA), on luminal A $(n=9)$ and TNBC tissue samples $(n=9)$. ACTB was used as a housekeeping gene for data normalization, both in the profiling and validation experiments. RNA extraction and cDNA synthesis were performed using the same kits as previously described, and cDNA was subsequently used for qPCR reactions in a 7900 HT Real-Time PCR System (Thermo Fisher Scientific, USA).

\subsection{Cell Lines}

Two BC cell lines representative for the two molecular subtypes were cultured under standard conditions. Mda-mb-231 (TNBC) and MCF7 (LuminalA) cells were collected upon confluence, and the individual expression of GAS5 and NEAT1 was measured as described above.

\subsection{Statistical Analysis}

The lncRNA expression level was calculated using the comparative $2^{-\Delta \Delta C T}$ method, relative to the selected controls [17]. Delta CT was calculated between the gene of interest and the reference gene ACTB. The CT cut-off was set to 37 .

We used Student's t-test to compare the relative lncRNA expression between the control group (Luminal A) and tested group (TNBC). The Mann-Whitney nonparametric test was used for cell line comparisons, given the small number of samples in each group. The cutoff value for statistical significance was set at $p<0.05$.

\section{Results}

The demographic characteristics of the patients, as well as the pathological features of the tumors are described in Table 1. The data are shown separately for TNBC and Luminal A groups.

The vast majority of the patients were older than 50 years in both groups $(77.78 \%$ and $88.89 \%$ in the TNBC and Luminal A group, respectively). More than half of the patients $(55.56 \%)$ had normal BMI values in both groups. Regarding the tumor characteristics, the patients presented in general tumor sizes smaller than $5 \mathrm{~cm}(55.56 \%$ for the TNBC group and $66.67 \%$ for the Luminal A group), from which more than half (55.56\%) were HER2 positive in both groups.

Table 2 shows the determined lncRNA species from the profiling step, with their corresponding fold changes and $p$ values. Only OTX2-AS1 and SOX2-OT had significant differences in gene expression. OTX2-AS1 had a fold change of $205.22(p=0.029)$, being overexpressed in TNBC tissues compared to luminal A, while SOX2-OT was downregulated, having a fold change of $0.02(p=0.042)$. In the validation step, none of these two lncRNAs presented statistical differences in gene expression (data not shown). 
Table 1. Patients' characteristics and pathological tumor features.

\begin{tabular}{|c|c|c|c|}
\hline Characteristics & & $\begin{array}{l}\text { TNBC } \\
N(\%)\end{array}$ & $\begin{array}{c}\text { Luminal A } \\
\quad N(\%)\end{array}$ \\
\hline \multirow{2}{*}{ Age } & $>50$ & 7 (77.78) & $8(88.89)$ \\
\hline & $<50$ & $2(22.22)$ & 1 (11.11) \\
\hline \multirow{2}{*}{ BMI } & Normal & $5(55.56)$ & $5(55.56)$ \\
\hline & Obese & $4(44.44)$ & $4(44.44)$ \\
\hline \multirow{2}{*}{ Stage } & I and II & $5(55.56)$ & $4(44.44)$ \\
\hline & III & $4(44.44)$ & $5(55.56)$ \\
\hline \multirow{2}{*}{ Tumor size } & $<5 \mathrm{~cm}$ & $5(55.56)$ & $6(66.67)$ \\
\hline & $>5 \mathrm{~cm}$ & $4(44.44)$ & $3(33.33)$ \\
\hline \multirow{2}{*}{$\begin{array}{l}\text { Lymph node } \\
\text { involvement }\end{array}$} & Yes & $5(55.56)$ & $4(44.44) *$ \\
\hline & No & $4(44.44)$ & $3(33.33) *$ \\
\hline \multirow[b]{2}{*}{ HER2 } & Positive & $5(55.56)$ & $5(55.56)$ \\
\hline & Negative & $4(44.44)$ & $4(44.44)$ \\
\hline \multirow{2}{*}{ Ki-67 } & Positive & $9(100.00)$ & $9(100.00)$ \\
\hline & Negative & $0(0.00)$ & $0(0.00)$ \\
\hline \multirow{2}{*}{ ER } & Positive & $0(0.00)$ & $9(100.00)$ \\
\hline & Negative & $9(100.00)$ & $0(0.00)$ \\
\hline \multirow{2}{*}{ PR } & Positive & $0(0.00)$ & $9(100.00)$ \\
\hline & Negative & $9(100.00)$ & $0(0.00)$ \\
\hline
\end{tabular}

* Percentages calculated from the total samples does not add up to $100 \%$ due to missing values (undetermined lymph node involvement for two patients in the Luminal A group).

Table 2. Differential expression of lncRNAs in the profiling study.

\begin{tabular}{cccccc}
\hline $\begin{array}{c}\text { LncRNA } \\
\text { Name }\end{array}$ & Fold Change & $p$ Value & $\begin{array}{c}\text { LncRNA } \\
\text { Name }\end{array}$ & Fold Change & $p$ Value \\
\hline BCYRN1 & 0.967 & 0.457 & MEG3 & 5.394 & 0.605 \\
BDNF-AS & 0.894 & 0.469 & NEAT1 & 5.818 & 0.548 \\
DISC2 & 0.624 & 0.325 & OTX2-AS1 & 205.222 & 0.029 \\
EGOT & 1.634 & 0.860 & PANDAR & 1.101 & 0.236 \\
FTX & 13.518 & 0.456 & PTENP1-AS & 0.989 & 0.708 \\
GACAT1 & 1.347 & 0.728 & SNHG16 & 1.115 & 0.614 \\
GAS5 & 1.734 & 0.914 & SOX2-OT & 0.020 & 0.042 \\
H19 & 1.163 & 0.764 & ST7-AS2 & 2.206 & 0.956 \\
HEIH & 1.388 & 0.702 & TERC & 1.652 & 0.812 \\
HOTAIR & 5.771 & 0.543 & TMEM161B- & 0.909 & 0.474 \\
IPW & 1.163 & 0.656 & TRERNA1 & 0.444 & 0.372 \\
JPX & 1.014 & 0.512 & TUG1 & 1.453 & 0.814 \\
KCNQ1OT1 & 4.028 & 0.690 & UCA1 & 0.386 & 0.601 \\
FALEC & 1.204 & 0.633 & XIST & 0.769 & 0.316 \\
LINC-ROR & 0.624 & 0.174 & ZFAS1 & 1.680 & 0.868 \\
MALAT1 & 1.763 & 0.936 & & & \\
\hline
\end{tabular}

Although GAS5 and NEAT1 did not reach statistical significance in the profiling step, we performed an individual validation for these two lncRNAs as they are highly associated with BC. GAS5 showed to be significantly downregulated in TNBC tissue samples compared to Luminal A tissue samples, and it was also downregulated in Mdamb-231 cell line compared to MCF7 cells. NEAT1 was also downregulated in both sample types of TNBC versus Luminal A; however, the comparison reached statistical significance only for cell lines, as shown in Table 3 and Figure 1a-d, respectively. 
Table 3. Differential expression of long non-coding RNAs (lncRNAs) in triple negative breast cancer (TNBC) versus Luminal A samples in the validation study.

\begin{tabular}{cccc}
\hline LncRNA & Sample Type & Fold Change & $p$ Value \\
\hline \multirow{2}{*}{ GAS5 } & FFPE tissue & 0.33 & 0.022 \\
& Cell lines & 0.52 & 0.035 \\
\hline \multirow{2}{*}{ NEAT1 } & FFPE tissue & 0.66 & 0.27 \\
& Cell lines & 0.23 & 0.035 \\
\hline
\end{tabular}

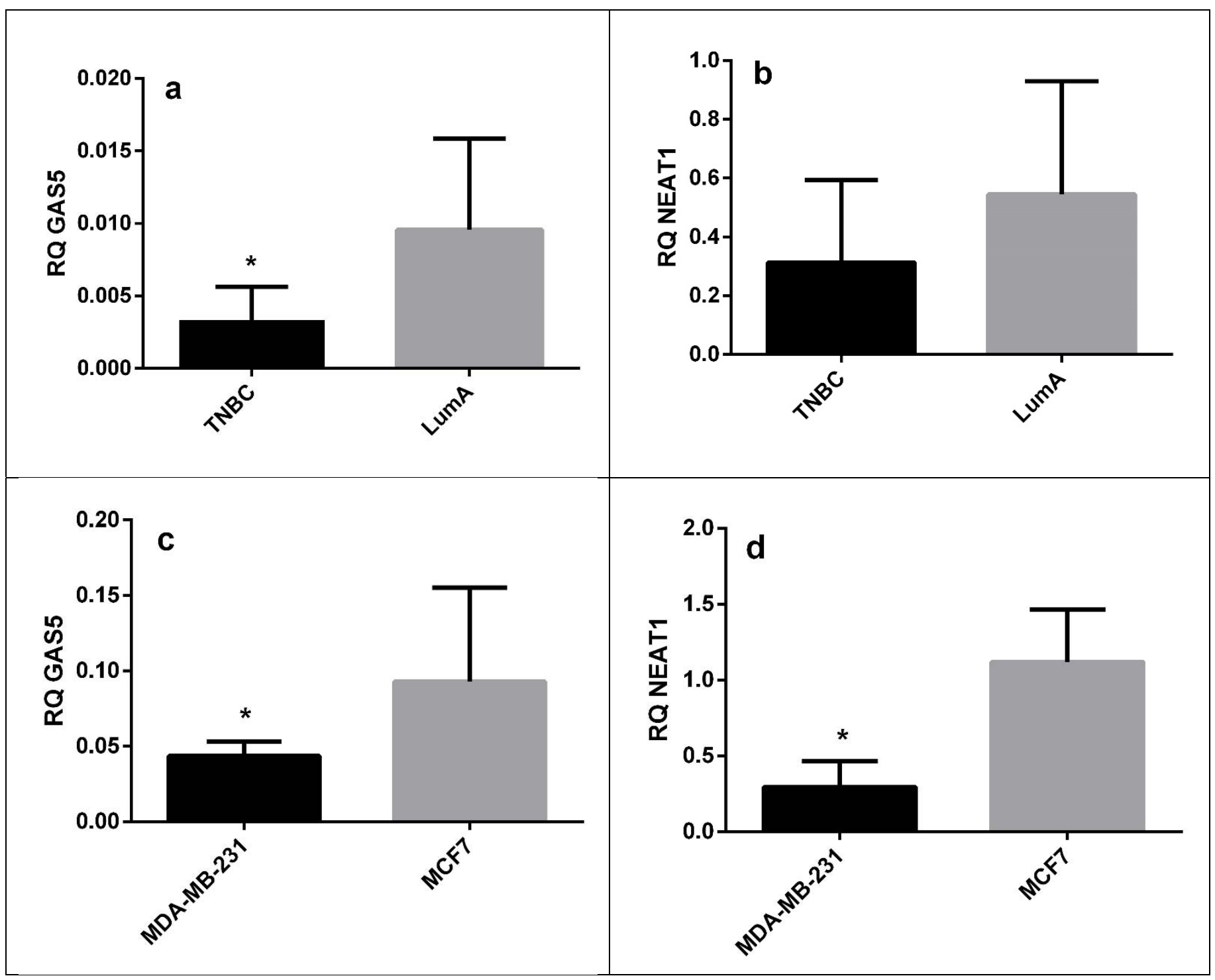

Figure 1. Relative quantity (RQ) of GAS5 (a) and NEAT1 (b) in the TNBC and Luminal A formalin-fixed paraffin-embedded (FFPE) samples and cell lines $(\mathbf{c}, \mathbf{d}) ;{ }^{*}$ denotes $p<0.05$.

\section{Discussion}

To our knowledge, this is the first study that assesses the differential expression of GAS5 IncRNA in different BC tissue subtypes using LCM technology. Therefore, we proved that LCM is a powerful tool that is capable of isolating specific, tumor-enriched regions of interest from archived (FFPE) tissue samples for a more accurate lncRNA profiling. The aim of our study was also to investigate potential differences in the expression levels 
of lncRNAs in two different tissue subtypes (TNBC and luminal A), as tissue-specific biomarkers for BC.

Our findings showed that two lncRNAs (OTX2-AS1 and SOX2-OT) were differentially expressed $(p<0.05)$ in TNBC tissues compared to luminal A; however, they were not subsequently validated. Previous literature studies demonstrated that the pluripotencyassociated transcription factor SOX2 gene is expressed and associated with at least 25 types of cancer, including BC [18]. Amaral et al. (2009) showed that the SOX2 gene is found in an intron of the multi-exon SOX2-OT lncRNA, and therefore, SOX2-OT acts as a transcriptional enhancer for the SOX2 gene [19].

However, little is known about the role of OTX2-AS1 in BC. In our study, OTX2-AS1 was also differentially expressed $(p<0.05)$ in TNBC tissues when compared to Luminal A samples in the profiling analysis.

In addition, as lncRNA clustering with mRNA PAM50 classification is tightly correlated [20,21], our study underwent in the same direction, proving that some IncRNA species are differentially expressed in TNBC tissues when compared to luminal A tissue samples.

Furthermore, we selected for validation two other individual lncRNAs that were previously associated with $\mathrm{BC}$, the growth arrest-specific transcript 5 (GAS5) and the nuclear enriched abundant transcript 1 (NEAT1), as they are two of the most studied lncRNAs in BC [22-26]. A study conducted by Li et al. (2018) reported that GAS5 levels are decreased in TNBC types, showing its potential role as a tumor suppressor. The ectopic expression of GAS5 in TNBC tissue samples showed an enhanced apoptosis and reduced proliferation of TNBC cells [22]. In order to enhance the tumor suppressor role of GAS5, Pickard et al. (2016) showed that GAS5 oligonucleotides could serve as therapeutic targets in $\mathrm{BC}$, as the hormone response element mimic (HREM) sequence within GAS5 alone can promote apoptosis of BC cells in the same manner as does the lncRNA GAS5 in full-length [27]. Our study corroborated with previous literature findings, GAS5 being significantly downregulated in TNBC tissues compared to luminal A tissues, as well as in the Mda-mb-231 cell line compared to MCF7 cells. The downregulation of GAS5 was correlated with a more aggressive phenotype [22], as TNBC subtype is hormone-receptor and HER2 negative and frequently associated with BRCA1 gene mutations, while luminal A is hormone-receptor positive, HER2 negative with low protein Ki-67 levels, thus having the best prognosis [5].

NEAT1 was also downregulated in TNBC cases in our study, although it did not reach statistical significance in tissue samples. Several reports have linked the abnormal expression of NEAT1 with BC, suggesting that the expression level of NEAT1 was significantly upregulated (6.86-fold increase) in TNBC tissues when compared to normal controls. The same report conducted by Shin et al. (2019) showed that NEAT1 promoted drug resistance and cancer stemness, and that NEAT1 knockdown minimized cell growth in cisplatin- or taxol-treated cells. NEAT1 silencing also decreased the tumor growth in vivo, suggesting therefore its oncogenic role [28]. Moreover, it was shown that NEAT1 is a direct transcriptional target of p53, and that the low expression of lncRNA NEAT1 in TNBC tissues is associated with poor prognosis of BC [29].

NEAT1 was also found to be significantly $(p=0.035)$ downregulated in our cell lines study in TNBC cells versus Luminal A, which is inconsistent with other literature studies. Previously, it was found that NEAT1 expression level is significantly upregulated in BC cell lines when compared to normal MCF-10A cells [30]. However, in our study, we used Luminal A cells (MCF7) as controls. Another report found NEAT1 levels as being upregulated in BC cells compared to normal cells, and that NEAT1 levels are inversely correlated with miR-133b, which in turn promotes BC migration and invasion [31].

Taken together, we suggest that GAS5 could be used as a tissue-specific diagnostic biomarker for $\mathrm{BC}$, as our findings showed a significant downregulation in TNBC compared to luminal A. Furthermore, we also suggest from our study that sample specimens of such heterogenous malignancies should be collected using a more prudent technique such as 
LCM technology, so as to limit the diversity of tissue specimen and precisely select the desired area of interest from samples.

Our study had some limitations that must be acknowledged, which arose primarily from the relatively low sample size and unmatched characteristics of the compared groups; therefore, validation in large-scaled powered studies is warranted. Secondly, our study design did not include a comparison between fixed and non-fixed tissue sample to test the limitations of the FFPE extraction step. However, the available literature reports comparing paired fresh and FFPE tissues show that even though RNA integrity can decrease during FFPE extraction, reproducible and highly correlated gene expression data can be obtained from FFPE samples of various tissue types, including breast cancer subtypes [32-35]. In addition, the use of more than one representative cell line for each breast cancer subtype would have been ideal.

Although we did prove that some lncRNAs possess tissue-specificity in matters of BC and that they are able to significantly distinguish between tissue subtypes, future studies of larger cohort sizes are needed to confirm our findings with higher confidence.

\section{Conclusions}

BC still remains a public health concern due to its complexity and heterogeneity. LncRNAs show great potential in deciphering the molecular mechanisms of this malignancy. Moreover, it is suggested that lncRNAs could be used as diagnostic and prognostic biomarkers for breast carcinoma because of their differential expression in the various tissue subtypes. Our study showed that the expression level of GAS5 is significantly downregulated in TNBC tissues and cell lines compared to Luminal A, suggesting a promising potential role as a tissue-specific diagnostic biomarker for BC. Nevertheless, more in-depth studies with larger sample sizes are required in order to better investigate lncRNAs tissue specificity, as well as their molecular mechanisms in the biology of BC.

Author Contributions: A.M. and D.N. collected, analyzed and interpreted the data, performed qPCR analysis, wrote, corrected, and formatted the manuscript. A.V. performed the LCM procedure, analyzed and interpreted the data and participated in manuscript writing. F.B. performed the histopathology diagnosis, IHC analysis, analyzed and interpreted the data and participated in manuscript writing. E.S. performed the LCM procedure, qPCR analysis, analyzed and interpreted the data and participated in manuscript writing. N.C. and R.I. collected the surgical tissue samples and patient clinical data, obtained informed consent and participated in manuscript writing. D.C. and C.A.D. performed the cell lines analysis, analyzed and interpreted the data, participated in manuscript writing. C.M. designed the study, analyzed and interpreted the data, performed the statistical analysis, wrote, corrected, reviewed, and formatted the manuscript. All authors have read and agreed to the published version of the manuscript.

Funding: The authors received no financial support for the current research.

Institutional Review Board Statement: Ethical review and approval were waived for this study, due to the fact that the samples used were older than 10 years and considered surplus diagnostic material.

Informed Consent Statement: Informed written consent was obtained from all subjects involved in the study at the date of sample collection.

Data Availability Statement: The data supporting the findings are available from the corresponding author, upon request.

Conflicts of Interest: The authors declare no potential competing interests for the current research, authorship and/or publication of this study.

Ethics Approval: The study was approved by the Ethical Committee of our institution.

Consent to Participate and for Publication: Informed consent was obtained from all patients before surgery for the use of their biological samples.

\section{References}

1. Siegel, R.L.; Miller, K.D.; Jemal, A. Cancer statistics. CA Cancer J. Clin. 2020, 70, 7-30. [CrossRef] 
2. The Cancer Genome Atlas Network., Genome sequencing centres: Washington University in St Louis., Koboldt, D.; et al. Comprehensive molecular portraits of human breast tumours. Nature 2012, 490, 61-70.

3. Nielsen, T.O.; Hsu, F.D.; Jensen, K.; Cheang, M.; Karaca, G.; Hu, Z.; Hernandez-Boussard, T.; Livasy, C.; Cowan, D.; Dressler, L.; et al. Immunohistochemical and clinical characterization of the basal-like subtype of invasive breast carcinoma. Clin. Cancer Res. 2004, 10, 5367-5374. [CrossRef] [PubMed]

4. Blows, F.M.; Driver, K.E.; Schmidt, M.K.; Broeks, A.; van Leeuwen, F.E.; Wesseling, J.; Cheang, M.C.; Gelmon, K.; Nielsen, T.O.; Blomqvist, C.; et al. Subtyping of breast cancer by immunohistochemistry to investigate a relationship between subtype and short and long term survival: A collaborative analysis of data for 10,159 cases from 12 studies. PLoS Med. 2010, 7, e1000279. [CrossRef] [PubMed]

5. Almarzooq, R.; Alrayes, A.; Alaradi, H.; Abdulla, H. Molecular subtypes of breast cancer. Bahrain Med Bull. $2018,40,222-225$.

6. Klopfleisch, R.; Weiss, A.T.; Gruber, A.D. Excavation of aburied treasure DNA, mRNA, miRNA and protein analysis in formalin fixed, paraffin embedded tissues. Histol. Histopathol. 2011, 26, 797-810.

7. Mathias, C.; Zambalde, E.P.; Rask, P.; Gradia, D.F.; de Oliveira, J.C. Long non-coding RNAs differential expression in breast cancer subtypes: What do we know? Clin. Genet. 2019, 95, 558-568. [CrossRef]

8. Johnsson, P.; Lipovich, L.; Grander, D.; Morris, K.V. Evolutionary conservation of long non-coding RNAs; sequence, structure, function. Biochim. Biophys. Acta (BBA) Gen. Subj. 2014, 1840, 1063-1071. [CrossRef]

9. Fu, P.F.; Zheng, X.; Fan, X.; Lin, A. Role of cytoplasmic lncRNAs in regulating cancer signaling pathways. J. Zhejiang Univ. Sci. B 2019, 20, 1-8. [CrossRef]

10. Chen, C.; Li, Z.; Yang, Y.; Xiang, T.; Song, W.; Liu, S. Microarray expression profiling of dysregulated long non-coding RNAs in triple-negative breast cancer. Cancer Biol. Ther. 2015, 16, 856-865. [CrossRef]

11. Augoff, K.; Mccue, B.; Plow, E.F.; Sossey-Alaoui, K. miR-31 and its host gene lncRNA LOC554202 are regulated by promoter hypermethylation in triple-negative breast cancer. Mol. Cancer 2012, 11, 1-13. [CrossRef]

12. Merry, C.R.; McMahon, S.; Forrest, M.E.; Bartels, C.F.; Saiakhova, A.; Bartel, C.A.; Scacheri, P.C.; Thompson, C.L.; Jackson, M.W.; Harris, L.N.; et al. Transcriptome-wide identification of mRNAs and lincRNAs associated with trastuzumab-resistance in HER2-positive breast cancer. Oncotarget 2016, 7, 53230-53244. [CrossRef] [PubMed]

13. Espina, V.; Wulfkuhle, J.D.; Calvert, V.S.; VanMeter, A.; Zhou, W.; Coukos, G.; Geho, D.H.; Petricoin, E.F., III; Liotta, L.A. Laser-capture microdissection. Nat. Protoc. 2006, 1, 586-603. [CrossRef] [PubMed]

14. Nariţa, D.; Anghel, A.; Seclaman, E.; Ilina, R.; Cireap, N.; Ursoniu, S. Molecular profiling of ADAM12 gene in breast cancers. Rom. J. Morphol. Embryol. 2010, 51, 669-676.

15. Seclaman, E.; Narita, D.; Anghel, A.; Cireap, N.; Ilina, R.; Sirbu, I.O.; Marian, C. MicroRNA Expression in Laser Micro-dissected Breast Cancer Tissue Samples-A Pilot Study. Pathol. Oncol. Res. 2019, 25, 233-239. [CrossRef]

16. Mihala, A.; Alexa, A.A.; Samoilă, C.; Dema, A.; Vizitiu, A.C.; Anghel, A.; Tămaş, L.; Marian, C.V.; Sîrbu, I.O. A pilot study on the expression of microRNAs resident on chromosome 21 in laser microdissected FFPE prostate adenocarcinoma samples. Rom. J. Morphol. Embryol. 2015, 56, 1063-1068. [PubMed]

17. Livak, K.J.; Schmittgen, T.D. Analysis of relative gene expression data using real-time quantitative PCR and the 2- $\Delta \Delta C T$ method. Methods 2001, 25, 402-408. [CrossRef] [PubMed]

18. Wuebben, E.L.; Rizzino, A. The dark side of SOX2: Cancer-A comprehensive overview. Oncotarget 2017, 8, 44917-44943. [CrossRef]

19. Amaral, P.P.; Neyt, C.; Wilkins, S.J.; Askarian-Amiri, M.E.; Sunkin, S.M.; Perkins, A.C.; Mattick, J.S. Complex architecture and regulated expression of the Sox2ot locus during vertebrate development. RNA 2009, 15, 2013-2027. [CrossRef]

20. Yan, X.; Hu, Z.; Feng, Y.; Hu, X.; Yuan, J.; Zhao, S.D.; Zhang, Y.; Yang, L.; Shan, W.; He, Q.; et al. Comprehensive Genomic Characterization of Long Non-coding RNAs across Human Cancers. Cancer Cell 2015, 28, 529-540. [CrossRef] [PubMed]

21. Zeng, Y.; Wang, G.; Zhou, C.F.; Zhang, H.B.; Sun, H.; Zhang, W.; Zhou, H.-H.; Liu, R.; Zhu, Y.-S. LncRNA Profile Study Reveals a Three-LncRNA Signature Associated with the Pathological Complete Response Following Neoadjuvant Chemotherapy in Breast Cancer. Front. Pharmacol. 2019, 10, 574. [CrossRef] [PubMed]

22. Li, S.; Zhou, J.; Wang, Z.; Wang, P.; Gao, X.; Wang, Y. Long noncoding RNA GAS5 suppresses triple negative breast cancer progression through inhibition of proliferation and invasion by competitively binding miR-196a-5p. Biomed. Pharmacother. 2018, 104, 451-457. [CrossRef] [PubMed]

23. Pickard, M.R.; Williams, G.T. Regulation of apoptosis by long non-coding RNA GAS5 in breast cancer cells: Implications for chemotherapy. Breast Cancer Res. Treat. 2014, 145, 359-370. [CrossRef]

24. Shin, V.Y.; Chen, J.; Cheuk, I.W.Y.; Siu, M.T.; Ho, C.W.; Wang, X.; Jin, H.; Kwong, A. Long non-coding RNA NEAT1 confers oncogenic role in triple-negative breast cancer through modulating chemoresistance and cancer stemness. Cell Death Dis. 2019, 10, 270. [CrossRef]

25. Jiang, X.; Zhou, Y.; Sun, A.J.; Xue, J.L. NEAT1 contributes to breast cancer progression through modulating miR-448 and ZEB1. J. Cell Physiol. 2018, 233, 8558-8566. [CrossRef] [PubMed]

26. Zhang, M.; Wu, W.B.; Wang, Z.W.; Wang, X.H. lncRNA NEAT1 is closely related with progression of breast cancer via promoting proliferation and EMT. Eur. Rev. Med. Pharmacol. Sci. 2017, 21, 1020-1026.

27. Pickard, M.R.; Williams, G.T. The hormone response element mimic sequence of GAS5 lncRNA is sufficient to induce apoptosis in breast cancer cells. Oncotarget 2016, 7, 10104-10116. [CrossRef] 
28. Idogawa, M.; Ohashi, T.; Sasaki, Y.; Nakase, H.; Tokino, T. Long non-coding RNA NEAT1 is a transcriptional target of p53 and modulates p53-induced transactivation and tumor-suppressor function. Int. J. Cancer 2017, 140, 2785-2791. [CrossRef]

29. Li, X.; Wang, S.; Li, Z.; Long, X.; Guo, Z.; Zhang, G.; Zu, J.; Chen, Y.; Wen, L. The lncRNA NEAT1 facilitates cell growth and invasion via the miR-211/HMGA2 axis in breast cancer. Int. J. Biol. Macromol. 2017, 105, 346-353. [CrossRef]

30. Li, X.; Deng, X.; Pang, X. LncRNA NEAT1 Silenced miR-133b Promotes Migration and Invasion of Breast Cancer Cells. Int. J. Mol. Sci. 2019, 20, 3616. [CrossRef]

31. Lv, Y.; Rao, Y.; Dong, W. Quantitative analysis of lncRNA from human FF and FFPE brain speimens. FASEB J. 2018, $32,525.1$.

32. Lv, Y.; Li, S.; Li, Z.; Tao, R.; Shao, Y.; Chen, Y. Quantitative analysis of noncoding RNA from paired fresh and formalin-fixed paraffin-embedded brain tissues. Int. J. Legal Med. 2020, 134, 873-884. [CrossRef] [PubMed]

33. Iddawela, M.; Rueda, O.M.; Klarqvist, M.; Graf, S.; Earl, H.M.; Caldas, C. Reliable gene expression profiling of formalin-fixed paraffin-embedded breast cancer tissue (FFPE) using cDNA-mediated annealing, extension, selection, and ligation whole-genome (DASL WG) assay. BMC Med. Genom. 2016, 9, 1-9. [CrossRef] [PubMed]

34. Zhang, P.; Lehmann, B.D.; Shyr, Y.; Guo, Y. The Utilization of Formalin Fixed-Paraffin-Embedded Specimens in High Throughput Genomic Studies. Int. J. Genom. 2017, 2017. [CrossRef] [PubMed]

35. Jovanović, B.; Sheng, Q.; Seitz, R.S.; Lawrence, K.D.; Morris, S.W.; Thomas, L.R.; Hout, D.R.; Schweitzer, B.L.; Guo, Y.; Pietenpol, J.A.; et al. Comparison of triple-negative breast cancer molecular subtyping using RNA from matched fresh-frozen versus formalin-fixed paraffin-embedded tissue. BMC Cancer 2017, 17, 1-14. 